\title{
Fixed point adjunctions for equivariant module spectra
}

\author{
J P C GREENLEES \\ BROOKE SHIPLEY
}

\begin{abstract}
We consider the Quillen adjunction between fixed points and inflation in the context of equivariant module spectra over equivariant ring spectra, and give numerous examples including some based on geometric fixed points and some on the Eilenberg-Moore spectral sequence.
\end{abstract}

55P42; 55N91, 55P91

\section{Introduction}

\subsection{Motivation}

We can view the present paper in two ways. On the one hand we can view it as an investigation of the formal properties of a basic change of groups adjunction in equivariant topology. On the other hand we can view it as giving a powerful context for proving two well-known general results. These are of course two sides of the same coin.

The change of groups adjunction is that between fixed points and inflation, placed in the more general context of modules over ring spectra, and will be introduced in the Section 1.2 below.

The two well-known results are usually considered in rather different contexts. The first (Sections 3 and 7) is the fact that the category of $G$-spectra lying over a normal subgroup $N$ is equivalent to the category of $G / N$-spectra. The second (Section 8 ) is the Eilenberg-Moore spectral sequence which states that (under suitable hypotheses) if $X$ is a free $G$-space, then $H^{*}(X)$ can be calculated from $H^{*}(X / G)$ as an $H^{*}(B G)$ module. Our versions of these results are both expressed as Quillen equivalences of model categories, and it is striking that these two things are rather formal consequences of a single formal statement together with the cellularization principle [15] (restated here as Theorem 2.1). We give a variety of other interesting specializations of the general results.

Particular instances of our general result are central ingredients in our work $[12 ; 13]$ giving algebraic models for categories of rational equivariant spectra. 


\subsection{Context}

When group actions are under consideration there is often a valuable adjunction between passage to fixed points under a normal subgroup $N$ of the ambient group $G$ and inflation from the quotient group $G / N$.

As a first example, if we are considering vector spaces we obtain an adjunction in representation theory,

$$
G-\operatorname{Hom}\left(\inf _{G / N}^{G} X, Y\right) \cong G / N-\operatorname{Hom} / N-\operatorname{Hom}\left(X, Y^{N}\right),
$$

where $Y$ is a vector space with $G$ action, and $X$ is a vector space with $G / N$ action. The fixed-point functor $(\cdot)^{N}$ and the inflation functor $\inf _{G / N}^{G}$ require no further explanation in this case, but we will use this terminology in other cases where the functors may be less familiar. The adjunction extends to graded vector spaces, and to graded vector spaces with a differential (chain complexes). If we replace vector spaces by topological spaces the adjunction is an elementary statement in equivariant topology.

In these cases, we may choose model structures so that the adjunction is a Quillen adjunction, so there is an induced adjunction between homotopy categories

$$
\left[\inf _{G / N}^{G} X, Y\right]^{G} \cong\left[X, Y^{N}\right]^{G / N} .
$$

Moving towards cases of concern to us here, we consider categories of equivariant orthogonal spectra (Mandell and May [21]) as our model. The Lewis-May fixed-point adjunction [19]

$$
(\cdot)^{N}: G \text {-spectra } \longleftrightarrow G / N \text {-spectra }: \inf _{G / N}^{G}
$$

applies to orthogonal spectra [21, V.3.4], and it is a Quillen adjunction.

The principal purpose of the present paper is to consider a variation of this adjunction with categories of module spectra over ring spectra.

\subsection{The case at hand}

The Lewis-May categorical fixed-point functor on orthogonal spectra is lax symmetric monoidal (preserves smash products precisely, but not units) [21, V.3.8], so that if $R$ is a ring $G$-spectrum, $R^{N}$ is a ring $G / N$-spectrum.

The purpose of this paper is to consider the Quillen adjunction

$$
\Psi^{N}: R-\bmod -G-\text { spectra } \longleftrightarrow R^{N}-\bmod -G / N-\text { spectra }: R \otimes_{\inf R^{N}}(\cdot)
$$


(here and elsewhere we follow Quillen in putting the left adjoint arrow on top when describing adjunctions). The existence of this follows from the general discussion on the interaction of model categories and monoidal structures of Schwede and the second author [23]. Indeed, in our case inflation is strong symmetric monoidal [21, V.1.5(v)], and where the left adjoint has this property, the functors on spectra induce a Quillen pair relating the categories of modules. We have used the notation $\Psi^{N}$ for the Lewis-May fixed points to highlight the change of ambient ring from $R$ to $R^{N}$.

It turns out that this is rather a rich context, and we make explicit a number of examples where the adjunction gives a very close relationship between the categories in a number of interesting cases. The key to this is the cellularization principle [15], which gives a language to describe the deviation from equivalence. The examples include spectra and modules concentrated over $N$ (giving the classical geometric fixed point equivalence) in Sections 3 and 7, Eilenberg-Mac Lane spectra in Section 5, sphere spectra in Section 6 and cochains on a free $G$-space (giving the Eilenberg-Moore theorem) in Section 8.

\subsection{Relationship to other results}

We proved these results during our work on algebraic models for rational torusequivariant spectra, and special cases appeared in the preprint [13]. Since they are of wider interest, we present them separately here and refer to [12] and [14] for these applications.

Acknowledgements The first author is grateful for support under EPSRC grant number EP/H040692/1. This material is based upon work by the second author supported by the National Science Foundation under grant number DMS-1104396.

\section{Preliminaries}

\subsection{The cellularization principle}

A key ingredient in applications is the cellularization principle of [15], which states that a Quillen adjunction induces a Quillen equivalence between cellularized stable model categories in the sense of Hirschhorn [17] provided the cells are small and the unit and counit are equivalences on cells. This is analogous to the statement that a natural transformation of cohomology theories that induces an isomorphism on spheres is an equivalence.

Theorem 2.1 (The cellularization principle [15, Theorem 2.7]) Let $\mathbb{M}$ and $\mathbb{N}$ be right proper, stable, cellular model categories and $F: \mathbb{M} \rightarrow \mathbb{N}$ a left Quillen functor with right adjoint $U$. Let $Q$ be a cofibrant replacement functor in $\mathbb{M}$ and $R$ a fibrant replacement functor in $\mathbb{N}$. 
(1) Let $\mathcal{K}=\left\{A_{\alpha}\right\}$ be a set of objects in $\mathbb{M}$ with $F Q \mathcal{K}=\left\{F Q A_{\alpha}\right\}$ the corresponding set in $\mathbb{N}$. Then $F$ and $U$ induce a Quillen adjunction

$$
F: \mathcal{K} \text {-cell-M } \longleftrightarrow F Q \mathcal{K}-\text { cell }-\mathbb{N}: U
$$

between the $\mathcal{K}$-cellularization of $\mathbb{M}$ and the $F Q \mathcal{K}$-cellularization of $\mathbb{N}$.

(2) If $\mathcal{K}=\left\{A_{\alpha}\right\}$ is a stable set of small objects in $\mathbb{M}$ such that for each $A$ in $\mathcal{K}$ the object FQA is small in $\mathbb{N}$ and the derived unit $Q A \rightarrow U R F Q A$ is a weak equivalence in $\mathbb{M}$, then $F$ and $U$ induce a Quillen equivalence between the cellularizations

$$
\mathcal{K} \text {-cell- } \mathbb{M} \simeq Q F Q \mathcal{K}-\text { cell- } \mathbb{N} .
$$

(3) If $\mathcal{L}=\left\{B_{\beta}\right\}$ is a stable set of small objects in $\mathbb{N}$ such that for each $B$ in $\mathcal{L}$ the object $U R B$ is small in $\mathbb{M}$ and the derived counit $F Q U R B \rightarrow R B$ is a weak equivalence in $\mathbb{N}$, then $F$ and $U$ induce a Quillen equivalence between the cellularizations

$$
U R \mathcal{L}-\text { cell- } \mathbb{M} \simeq Q \mathcal{L}-\text { cell }-\mathbb{N}
$$

\subsection{Universal $G$-spaces}

If $\mathcal{K}$ is a family of subgroups (ie, a set of subgroups closed under conjugation and passage to smaller subgroups), there is a universal space $E \mathcal{K}$, characterized up to equivalence by the fact that $(E \mathcal{K})^{H}$ is empty if $H \notin \mathcal{K}$ and is contractible if $H \in \mathcal{K}$. We write $\widetilde{E} \mathcal{K}$ for the unreduced suspension $S^{0} * E \mathcal{K}$, so that there is a cofibre sequence

$$
E \mathcal{K}_{+} \longrightarrow S^{0} \longrightarrow \tilde{E} \mathcal{K} .
$$

For normal subgroups $N$ we define certain spaces $E\langle N\rangle$ by the cofibre sequence

$$
E[\subset N]_{+} \longrightarrow E[\subseteq N]_{+} \longrightarrow E\langle N\rangle .
$$

We note that in fact $E\langle N\rangle \simeq E G / N_{+} \wedge \widetilde{E}[\nsupseteq N]$.

\subsection{Geometric isotropy}

Recall that the geometric fixed-point functor extends the fixed-point space functor in the sense that for based $G$-spaces $Y$ there is a $G / N$-equivalence $\Phi^{N} \Sigma^{\infty} Y \simeq \Sigma^{\infty}\left(Y^{N}\right)$. The isotropy groups of a based $G$-space are the subgroups so that the fixed points are nontrivial, so it is natural to consider a stable, homotopy invariant version: the geometric isotropy of a $G$-spectrum $X$ is defined by

$$
\mathcal{G I}(X):=\left\{K \mid \Phi^{K} X \not{*} *\right\}
$$


Certain $G$-spaces are useful in picking out isotropy information. The geometric isotropy of $E \mathcal{F}_{+}$is $\mathcal{F}$ and the geometric isotropy of $\widetilde{E} \mathcal{F}$ is $\mathcal{F}^{c}$, the complement of $\mathcal{F}$.

\subsection{Geometric fixed points}

We say that $X$ lies over $N$ if every subgroup in $\mathcal{G I}(X)$ contains $N$. It is obvious for spaces $X$ that the inclusion $X^{N} \rightarrow X$ induces an equivalence $X^{N} \wedge \widetilde{E}[\nsupseteq N] \simeq$ $X \wedge \widetilde{E}[\nsupseteq N]$ and with a little care there is a generalization to the case when $X$ is a spectrum.

The relationship between categorical fixed points and geometric points is described in [19, II.9]. It follows from the geometric fixed-point Whitehead theorem that $X$ lies over $N$ if and only if

$$
X \simeq X \wedge \widetilde{E}[\nsupseteq N]
$$

and that if $Y$ lies over $N$ then

$$
[X, Y]^{G} \cong\left[\Phi^{N} X, \Phi^{N} Y\right]^{G / N} .
$$

\section{Models of spectra over $N$}

Some of the results in this section are well known, and we record them here partly to prepare the way for their module counterparts in Section 7.

\subsection{Models via localization}

We recall two models for the homotopy category of spectra over $N$ and their equivalence.

Choose a $G$-space $\widetilde{E}[\nsupseteq N]$, and without change in notation we take a bifibrant replacement of its suspension spectrum. We consider the class $\Phi^{N}$-equiv of morphisms $f: X \rightarrow Y$ so that $\widetilde{E}[\nsupseteq N] \wedge f$ is an equivalence; these are called equivalences over $N$.

Construction 3.1 [21, IV.6] We form the local model as the (left) Bousfield localization

$$
G \text {-spectra } / N:=L_{\Phi^{N} \text {-equiv }} G \text {-spectra. }
$$

The weak equivalences are the equivalences over $N$ and the cofibrations are those in the underlying category of $G$-spectra.

The inflation functor and its right adjoint, the Lewis-May fixed-point functor, are familiar in this context. 
Proposition 3.2 [21, VI.5.3] Composing the Quillen adjunction

$$
(\cdot)^{N}: G \text {-spectra } \longleftrightarrow G / N \text {-spectra }: \inf
$$

with localization, we obtain a Quillen equivalence

$$
G \text {-spectra/ } N \simeq G / N \text {-spectra. }
$$

\subsection{Models via modules}

There is a tempting approach to the category of spectra over $N$ by viewing them as modules over a ring. However, there is some need for caution.

First note that $\tilde{E}[\nsupseteq N]$ is a ring spectrum up to homotopy. A $G$-spectrum is an $\widetilde{E}[\nsupseteq N]$-module up to homotopy if and only if it lies over $N$. Similarly, any map of $G$-spectra over $N$ is compatible up to homotopy with the homotopy multiplication of $\widetilde{E}[\nsupseteq N]$. Thus the category of $\widetilde{E}[\nsupseteq N]$-modules looks like another model for the category of $G$-spectra over $N$. The homotopy category of this category of modules is not obviously triangulated.

However we can tighten up the structure. To begin, construct $\widetilde{E}[\nsupseteq N]$ as a localization of the ring spectrum $S^{0}$. Accordingly, it admits the structure of an associative ring $G$ spectrum (Elmendorf, Kriz, Mandell and May [5]) and we may consider its category of modules. Denote restriction of scalars along the map of ring spectra $l: S^{0} \rightarrow \widetilde{E}[\nsupseteq N]$ by $l^{*}$. Its right adjoint is the coextension of scalars functor

$$
l_{!}(M)=F(\widetilde{E}[\nsupseteq N], M) .
$$

Together these give a Quillen adjunction

$$
l^{*}: \tilde{E}[\nsupseteq N]-\bmod -G-\text { spectra } \rightleftarrows S^{0}-\bmod -G-\text { spectra }: l_{\text {! }}
$$

of module categories. This provides another model for $G$-spectra over $N$.

Proposition 3.3 The restriction and coextension of scalars functors induce a Quillen equivalence

$$
\tilde{E}[\nsupseteq N]-\bmod -G-\text { spectra } \simeq G-\text { spectra } / N \text {. }
$$

Proof Since the left Quillen functors are in the right order to be composed, we need only check that for any cofibrant $\widetilde{E}[\nsupseteq N]$-module $C$ and any $G$-spectrum $L$, fibrant in $G$-spectra over $N$, that a map $C \rightarrow l_{!} L$ is an equivalence in $\widetilde{E}[\nsupseteq N]$-modules if and only if $C \rightarrow L$ is an equivalence over $N$. Decoding this, we need to show that, in the ambient category of $G$-spectra, $C \rightarrow F(\tilde{E}[\nsupseteq N], L)$ is an equivalence if and only if $C \wedge \widetilde{E}[\nsupseteq N] \rightarrow L \wedge \widetilde{E}[\nsupseteq N]$ is an equivalence. 
Consider the following diagram:

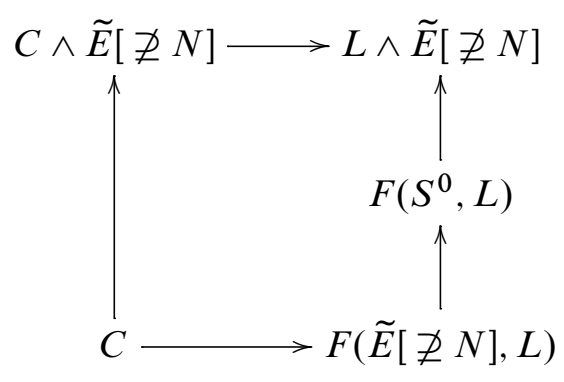

Since $C$ is a cofibrant $\widetilde{E}[\nsupseteq N]$-module, the left hand vertical is an equivalence. Since $L$ is fibrant, [21, IV.6.13] shows the right hand verticals are both equivalences. This gives the desired statement that the top horizontal is an equivalence if and only if the bottom horizontal is an equivalence.

The disappointment is that although $\widetilde{E}[\nsupseteq N]$ is a commutative ring up to homotopy, no model for it is a strictly commutative ring for reasons described by McClure [22] (or because it is incompatible with the existence of multiplicative norm maps). These phenomena are studied systematically by Hill and Hopkins in [16].

\section{Fixed point equivalences}

In this section we explain how to apply the cellularization principle to obtain interesting equivalences from the fixed-point adjunction

$$
\Psi^{N}: R-\bmod -G-\text { spectra } \longleftrightarrow R^{N}-\bmod -G / N-\operatorname{spectra}: R \otimes_{\inf R^{N}}(\cdot)
$$

on module categories.

\subsection{Towards Quillen equivalences}

In somewhat simplified notation, consider the unit and counit of the derived adjunction

$$
\eta: Y \longrightarrow\left(R \otimes_{\inf R^{N}} \inf Y\right)^{N} \quad \text { and } \quad \epsilon: R \otimes_{\inf R^{N}}\left(\inf X^{N}\right) \longrightarrow X .
$$

We see that $\eta$ is an equivalence for $Y=R^{N}$ and that $\epsilon$ is an equivalence for $X=R$, so that by the cellularization principle we always have an equivalence

$$
R \text {-cell- } R-\bmod -G \text {-spectra } \simeq R^{N}-\text { cell- } R^{N}-\bmod -G / N \text {-spectra. }
$$

If $G$ is trivial, $R$ generates the category of $R$-modules, but if $G$ is not trivial, $R$ is not usually a generator of the category of equivariant $R$-modules since we also need 
the modules of the form $R \wedge G / H_{+}$for proper subgroups $H$. Similar remarks apply to $R^{N}$-modules when $G / N$ is nontrivial.

\subsection{Thick category arguments}

Having established the interest in when categories of equivariant modules are generated by the ring, we need some more terminology.

If $R$ does build every $R$-module, we refer to the module category as monogenic. There are surprisingly many examples of monogenic equivariant module categories, and in practice the process of building the modules $R \wedge G / H_{+}$is of a simple form.

Definition 4.1 (i) Two $G$-spectra $X$ and $Y$ are $R$-equivalent $\left(X \sim_{R} Y\right.$ ) if there is an equivalence $R \wedge X \simeq R \wedge Y$ of $R$-modules.

(ii) A $G$-spectrum $X$ is an $R$-retract of $Y$ if $R \wedge X$ is a retract of $R \wedge Y$ as $R$-modules.

(iii) A collection $\mathcal{C}$ of $G$-spectra is closed under $R$-triangles if whenever there is a cofibre sequence $R \wedge X \rightarrow R \wedge Y \rightarrow R \wedge Z$ of $R$-modules then if two of $X, Y$ and $Z$ lie in $\mathcal{C}$, so does the third.

(iv) A class $\mathcal{C}$ of $G$-spectra is $R$-thick if it is closed under $R$-equivalence, $R$-retracts and completing $R$-triangles. We say that $X$ finitely $R$-builds $Y\left(X \models_{R} Y\right)$ if $Y$ is in the $R$-thick subcategory generated by $X$.

(v) A class $\mathcal{C}$ of $G$-spectra is $R$-localizing if it is $R$-thick and closed under arbitrary coproducts. We say that $X R$-builds $Y\left(X \vdash_{R} Y\right)$ if $Y$ is in the $R$-localizing subcategory generated by $X$.

(vi) We say that the category of $R$-modules is strongly monogenic if the $R$-localizing subcategory generated by $S^{0}$ is the entire category of $G$-spectra.

We note that to show the category of $R$-modules is strongly monogenic, we need only show that $G / H_{+}$is $R$-built by $S^{0}$ for all subgroups $H$. The following observation explains the purpose of the definitions.

Lemma 4.2 If the category of $R$-modules is strongly monogenic, then $R$-cellularization has no effect on $R-\bmod -G$-spectra, and we have a Quillen equivalence

$$
R-\text { cell }-R-\bmod -G-\text { spectra } \simeq R-\bmod -G-\text { spectra } .
$$


Proof By the work of Schwede and the second author [24, 2.2.1], the category of $R$-modules is strongly monogenic if and only if $R$ detects weak equivalences. Thus, here the $R$-cellular equivalences and the underlying equivalences agree.

Combining this with the fixed-point $R$-module Quillen adjunction from Section 4.1, we obtain a statement we will use repeatedly.

Corollary 4.3 If the category of $R$-modules is strongly monogenic

$$
R-\bmod -G-\text { spectra } \simeq R^{N} \text {-cell- } R^{N}-\bmod -G / N-\text { spectra }
$$

and if in addition $R^{N}$ is strongly monogenic (for example if $N=G$ ), then

$$
R-\bmod -G-\text { spectra } \simeq R^{N}-\bmod -G / N-\text { spectra. }
$$

\subsection{Strongly monogenic examples}

Perhaps the first important example of $R$-equivalence is given by Thom isomorphisms: if $R$ is complex orientable then it is complex stable, that is, for any complex representation $V$, we have $R \wedge S^{V} \simeq R \wedge S^{|V|}$. So, in particular, $S^{V} \sim_{R} S^{|V|}$, where $|V|$ denotes the underlying vector space of $V$ with trivial $G$-action.

Lemma 4.4 If $G$ is a torus and $R$ is complex stable then the category of $R$-modules is strongly monogenic.

Proof It suffices to show $S^{0} R$-builds all spectra $G / H_{+}$.

The proof is built from one cofibre sequence for the circle group and the Thom isomorphism for $R$. Suppose first that $\alpha: G \rightarrow U(1)$ is a nontrivial representation, and write $\bar{G}=G / K$ where $K=\operatorname{ker}(\alpha)$ for the relevant circle quotient, noting that there is an equivariant homeomorphism $S(\alpha)=\bar{G}$. The important cofibre sequence is

$$
\bar{G}_{+}=S(\alpha)_{+} \longrightarrow S^{0} \longrightarrow S^{\alpha} .
$$

The Thom isomorphism shows $S^{\alpha} \sim_{R} S^{2}$, and the cofibre sequence shows $\bar{G}_{+}$is in the $R$-thick subcategory generated by $S^{0}$.

The subgroups occurring as kernels $K$ as in the previous paragraph are precisely those of the form $K=H \times C$, where $H$ is a rank- $(r-1)$ torus and $C$ is a finite cyclic group. Finally we note that for an arbitrary subgroup $L$ we have

$$
G / L=G / K_{1} \times G / K_{2} \times \cdots \times G / K_{r}
$$

for suitable $K_{i}$ occurring as kernels.

Using the above argument up to $r$ times we see $G / L_{+}$is in the $R$-thick subcategory generated by $S^{0}$. 
When $G$ is not a torus we need additional restrictions to permit a similar argument. The flavour is that the group acts trivially on the coefficients, and there are many examples starting with mod $p$ Borel cohomology when $G$ is a $p$-group.

Lemma 4.5 If $G$ is a 2-group and for all subgroups $H$ of $G, R_{*}^{H}(\cdot)$ has Thom isomorphisms for all real representations, then the category of $R$-modules is strongly monogenic.

Proof The essential ingredients in the proof are a cofibre sequence for the group of order 2 and the Thom isomorphism for $R$.

We argue by induction on the order of $G$. The statement is immediate for the group of order 1, and we may suppose by induction that the statement has been proved for all proper subgroups of $G$.

Now consider $G$ itself. We must show that for all subgroups $L$ of $G$, the $G-$ space $G / L_{+}$is $R$-built from $R$. This is immediate if $L=G$, and in other cases we may choose a maximal subgroup $K$ of $G$ so that $L \subseteq K \subseteq G$. First we observe that by induction $G / L_{+}$is $R$-built from $G / K_{+}$. Indeed, $G / L_{+} \simeq G_{+} \wedge_{K} K / L_{+}$; by induction $K / L_{+}$is $K$-equivariantly $R$-built from $S^{0}$, and inducing up to $G$ we see $G / L_{+}$is $G$-equivariantly $R$-built from $G / K_{+}$. It remains to show that $G / K_{+}$ is $G$-equivariantly built from $S^{0}$.

Let $\xi: G \rightarrow O(1)$ be the representation with kernel $K$. Write $\bar{G}=G / K$ for the quotient of order 2 and note that there is an equivariant homeomorphism $S(\xi)=\bar{G}$. The important cofibre sequence is

$$
\bar{G}_{+}=S(\xi)_{+} \longrightarrow S^{0} \longrightarrow S^{\xi} .
$$

The Thom isomorphism shows $S^{\xi} \sim_{R} S^{1}$, and the first cofibre sequence shows $\bar{G}_{+}$ is in the $R$-thick subcategory generated by $S^{0}$. This shows $\bar{G}_{+}$is in the $R$-thick category generated by $S^{0}$ as required.

Lemma 4.6 If $G$ is a $p$-group, and for all subgroups $H$ of $G, R_{H}^{*}(\cdot)$ is complex stable, and $N_{G}(H)$ acts trivially on $R_{H}^{*}$ then the category of $R$-modules is strongly monogenic.

Proof The structure of the proof is precisely the same as for Lemma 4.5, and precisely as in that case, it suffices to show that $G / K_{+}$is $R$-built from $S^{0}$ for a maximal subgroup $K$. 
The difference is that we now need two cofibre sequences rather than just one. Suppose that $\alpha: G \rightarrow U(1)$ is a representation with kernel $K$. Write $\bar{G}=G / K$ for the quotient of order $p$. The first important cofibre sequence is

$$
S(\alpha)_{+} \longrightarrow S^{0} \longrightarrow S^{\alpha},
$$

and the second is the stable cofibre sequence

$$
\bar{G}_{+} \stackrel{1-g}{\longrightarrow} \bar{G}_{+} \longrightarrow S(\alpha)_{+},
$$

where $g$ is a suitable generator of $\bar{G}$. The Thom isomorphism shows $S^{\alpha} \sim_{R} S^{2}$, and the first cofibre sequence shows $S(\alpha)_{+}$is in the $R$-thick subcategory generated by $S^{0}$. Now since $\bar{G}$ acts trivially on $R_{*}^{K}$, we obtain a short exact sequence

$$
0 \longrightarrow R_{*}^{K} \longrightarrow R_{*}^{G}\left(S(\alpha)_{+}\right) \longrightarrow \Sigma R_{*}^{K} \longrightarrow 0 .
$$

Since the quotient is free over $R_{*}^{K}$, there is a splitting map $\Sigma R \wedge G / K_{+} \rightarrow R \wedge S(\alpha)_{+}$ of $R$-modules, and

$$
R \wedge S(\alpha)_{+} \simeq R \wedge \bar{G}_{+} \vee \Sigma R \wedge \bar{G}_{+} .
$$

This shows $\bar{G}_{+}$is in the $R$-thick category as required.

Remark 4.7 The action condition can be weakened somewhat, first since it is only necessary for elements of order $p$ in $W_{G}(K)$ to act trivially, and secondly we need only require $1-g$ to act nilpotently.

\section{Eilenberg-Mac Lane spectra}

In this section we consider the special case where the ambient ring spectrum represents ordinary cohomology, for which we need to recall some terminology. It is worth bearing in mind that for finite groups and rational coefficients this is the general case; see [24, 5.1.2] and the work of the first author and May [11, A.1].

\subsection{Mackey functors}

We recall that a $G$-Mackey functor is a contravariant additive functor $M: \mathcal{S O}_{G} \rightarrow$ AbGp on the stable orbit category $\mathcal{S O}_{G}$. For brevity, we write $M(H)$ for the value on $G / H_{+}$. When $G$ is finite, this is equivalent [19, V.9.9] to the classical definition of a collection of abelian groups $M(H)$ related by restrictions and transfers satisfying the Mackey formula; see Dress [3]. 
Evidently any $G$-equivariant cohomology theory $F$ gives rise to a graded Mackey functor $\underline{F}_{G}^{*}$ whose value at $H$ is $F_{H}^{*}$. Equivalently the homotopy groups of $F$ give a Mackey functor $\underline{\pi}_{*}^{G}(F)=[\cdot, F]_{*}^{G}$ whose value at $H$ is $\pi_{*}^{H}(F)=\left[G / H_{+}, F\right]_{*}^{G}$. A cohomology theory whose value on all homogeneous spaces is concentrated in degree 0 is called ordinary or Bredon. For any Mackey functor $M$, up to equivalence there is a unique cohomology theory with coefficient functor $M$, and we write $H M$ for the representing $G$-spectrum. Thus

$$
H_{G}^{*}(X ; M)=H M_{G}^{*}(X)=[X, H M]_{G}^{*} .
$$

The category of $G$-Mackey functors has a symmetric monoidal product which can be swiftly defined by

$$
M \square N=\underline{\pi}_{0}^{G}(H M \wedge H N),
$$

but this can be made categorically explicit using the Day coend construction; see Day [2] and Lewis [18].

A monoid in the category of Mackey functors is called a Green functor. If $\mathbb{R}$ is a Green functor $H \mathbb{R}$ is a ring spectrum, and if the Green functor is commutative, the ring spectrum can also be taken to be commutative; see Ullman [25]. A prime example is the representable Mackey functor $\mathbb{A}_{T}$, where $T$ is a disjoint union of orbits, with $\mathbb{A}_{T}(H)=\left[G / H_{+}, T_{+}\right]_{0}^{G}$. In particular the Burnside functor itself is the case when $T$ is a point and has $\mathbb{A}=\underline{\pi}_{0}^{G}\left(S^{0}\right)$, so that $\mathbb{A}(H)$ is the Burnside ring $A(H)$ of virtual $H$-sets.

\subsection{Modules over an Eilenberg-Mac Lane spectrum}

We are now ready to discuss $R=H \mathbb{R}$, where $\mathbb{R}$ is a Green functor, and we take $N=G$ so we can concentrate on the new features. In this case the fixed-point spectrum is also an Eilenberg-Mac Lane spectrum $R^{G}=(H \mathbb{R})^{G}=H(\mathbb{R}(G))$. We are considering the following adjunction:

$$
\Psi^{G}: H \mathbb{R}-\bmod -G-\text { spectra } \longleftrightarrow H \mathbb{R}(G) \text {-mod-spectra }: H \mathbb{R} \otimes_{H \mathbb{R}(G)}(\cdot)
$$

We can be a little more explicit. First note that for any connective nonequivariant spectrum $X$ we have $\underline{\pi}_{0}^{G}(\inf X)=\pi_{0}(X) \otimes \mathbb{A}$, where $\mathbb{A}$ is the Burnside functor. Indeed since Burnside rings are free over $\mathbb{Z}$, both sides are (Mackey functor valued, nonequivariant) homology theories of $X$; the universal property of $\mathbb{A}$ gives a natural transformation, and it is an isomorphism for $X=S^{0}$. Next, note that when $X$ is connective,

$$
[\inf X, H \mathbb{R}]^{G}=H \mathbb{R}_{G}^{0}(\inf X)=\operatorname{Hom}\left(\pi_{0}(X), \mathbb{R}(G)\right) .
$$


First note that both sides depend only on the 1-skeleton of $X$, and now it suffices to note that there is a natural comparison map which is an isomorphism for a wedge of copies of $S^{0}$, and both sides are left exact. It therefore follows that the counit of the adjunction

$$
\inf R^{G}=\inf H \mathbb{R}(G) \longrightarrow H \mathbb{R}=R
$$

is determined up to homotopy by being the scalar extension of the identity map in $\pi_{0}^{G}$. Note that if $G$ is finite $H \mathbb{R}_{*}^{K}\left(Y_{+}\right)$is concentrated in degree 0 for any subgroup $K$ and any finite $K$-set $Y$. Accordingly, $H \mathbb{R}$-module maps between the generators $G / H_{+} \wedge H \mathbb{R}$ of the category of $H \mathbb{R}$-modules are concentrated in degree 0 and Morita theory $[24,5.1 .1]$ shows that we have a purely algebraic description of the categories concerned:

$$
H \mathbb{R}-\bmod -G-\text { spectra } \simeq \mathbb{R}-\bmod -G-\text { Mackey },
$$

where 'mod' in the algebraic setting refers to differential graded modules. Thus we are considering

$$
\operatorname{ev}_{G}: \mathbb{R} \text {-mod-G-Mackey } \longleftrightarrow \mathbb{R}(G) \text {-mod-Abgrps }: \mathbb{R} \otimes_{\mathbb{R}(G)}(\cdot) .
$$

This is a Green ring analogue of one of the adjoints of evaluation, see the first author and May [10], and it would be interesting to give a more systematic account of the case $N \neq G$.

Remark 5.1 An instructive nonexample is to take the ring $R=\mathbb{Q}[G]$, so that $R^{N}=\mathbb{Q}[G / N]$. This is not an equivariant ring spectrum since, with the implied action of $G$ on $R$ by left translation, the multiplication on $R$ is not $G$-equivariant, and the unit map is not $G$-equivariant.

The alternative to this is to consider the conjugation action on $G$, and then we obtain a genuine equivariant ring. Of course this applies equally to the group ring $R=k\left[G^{c}\right]=$ $k \wedge G_{+}^{c}$ for any commutative ring spectrum $k$.

\section{The sphere spectrum}

Perhaps the first naturally occurring example has $R=S^{0}$ and $N=G$. We first observe that by the Segal-tom Dieck splitting we have

$$
R^{G}=\left(S^{0}\right)^{G} \simeq \bigvee B W_{G}(H)^{L(H)},
$$

where $L(H)$ is the representation on the tangent space to the identity coset of $G / H$. This is rather a complicated spectrum, and utterly different from the sphere. 
For simplicity we restrict attention to the rational case.

\subsection{Finite groups}

When $G$ is finite, and we work rationally, the sphere is the Eilenberg-Mac Lane spectrum for the rationalized Burnside functor $\mathbb{A}: S^{0} \simeq \mathbb{Q} H \mathbb{A}$, and this is a special case of the material in Section 5.2. In particular $R^{G}$ (which is a product of rational spheres) is more naturally described as the Eilenberg-Mac Lane spectrum for the rationalization of the Burnside ring $A(G)=\pi_{0}^{G}\left(S^{0}\right)=\pi_{0}\left(\left(S^{0}\right)^{G}\right)$. Thus we are considering the usual fixed-point adjunction and the adjunction between the Quillen equivalent algebraic categories from Section 5.2:

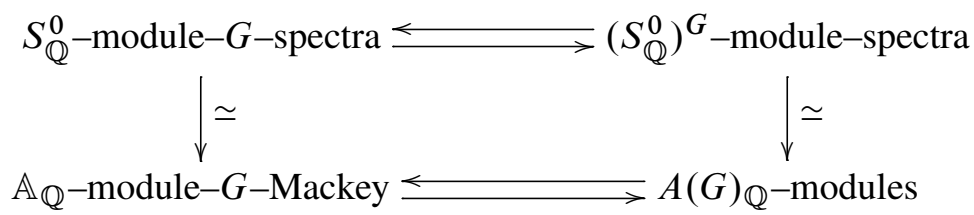

It is simplest to discuss the lower purely algebraic adjunction, but of course the discussion has a complete topological counterpart.

The mark homomorphism $A(G) \rightarrow \prod_{(H)} \mathbb{Z}$ is a rational isomorphism, so we have a complete set of idempotents $e_{H} \in A(G)_{\mathbb{Q}}$, and we may split both sides. On the right, by definition of the idempotents, $e_{H} A(G)_{\mathbb{Q}} \cong \mathbb{Q}$. On the left, evaluation at $G / H$ gives an isomorphism [11, Theorem A.9]

$$
e_{H} \mathbb{A}_{\mathbb{Q}} \text {-modules } \stackrel{\cong}{\longrightarrow} W_{G}(H) \text {-modules. }
$$

Picking one subgroup $H$ and considering just the $H^{\text {th }}$ factor by applying the idempotent $e_{H}$ to the four categories in the above diagram gives the top two lines in the next diagram. For the bottom line we then use the computations from this paragraph to simplify the algebraic categories:

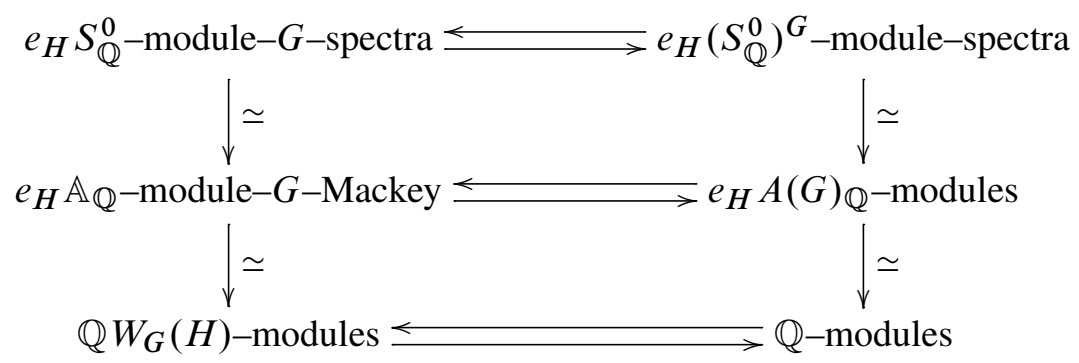

The right adjoint at the top is $G$-fixed points, in the middle it is evaluation at $G / G$ and at the bottom it $W_{G}(H)$-fixed points. 
If $W_{G}(H)$ is nontrivial, there is more than one simple $\mathbb{Q} W_{G}(H)$-module, so the $H^{\text {th }}$ factors on the left and right of the bottom row are inequivalent for most subgroups $H$. Indeed, in the bottom row the right adjoint takes $W_{G}(H)$-fixed points, so that on the right we have just the part corresponding to the trivial module. In model-theoretic terms, the cellularization principle shows that the category of $\mathbb{Q} W_{G}(H)$-modules with trivial action (equivalent to the category of $\mathbb{Q}$-modules) is the cellularization of the category of all $\mathbb{Q} W_{G}(H)$-modules with respect to $\mathbb{Q}$.

Reassembling the pieces by considering the product categories, we reach the conclusion that for $R=S_{\mathbb{Q}}^{0}$, the fixed-point inflation adjunction is

$$
R: \prod_{(H)} \mathbb{Q} W_{G}(H)-\bmod \longleftarrow \prod_{(H)} \mathbb{Q}-\bmod : L
$$

which is only an equivalence when $G$ is trivial.

\subsection{The circle group}

When $G$ is the circle group, the $\operatorname{ring} \pi_{*}^{G}\left(S^{0}\right)$ has trivial multiplication, so most information is captured in nonzero homological degree. In any case, the fixed-pointinflation adjunction is not a Quillen equivalence either (by connectivity the counit is not an equivalence for a free cell $G_{+}$). To get a Quillen equivalence with a category of modules over a nonequivariant ring spectrum it is more effective to use a Koszul dual approach as in Example 8.3 below, and as given in detail by the first author in [8] and by both authors in [13].

\section{Models of categories of modules over $N$}

A very satisfying class of examples is a generalization of the basic property of geometric fixed-point spectra. This takes the results in Section 3 into the context of modules over a ring spectrum.

To start we consider an arbitrary ring $G$-spectrum $R$ and introduce the notation

$$
\mathbb{C}_{0}=R \text {-module- } G \text {-spectra. }
$$

Now we may consider a model of $R$-module $G$-spectra over $N$, just as for the sphere in Section 3,

$$
\mathbb{C}_{1}=L_{\Phi^{N}}(R-\text { module- } G \text {-spectra }) .
$$

Now the $G / N$-spectrum $\bar{R}=\Phi^{N} R$ is the $N$-fixed points of the associative ring spectrum $R^{\prime}=R \wedge \widetilde{E}[\nsupseteq N]$, and hence it is an associative ring with a module category

$$
\mathbb{C}_{2}=\Phi^{N} R \text {-module- } G / N \text {-spectra. }
$$


If $\bar{R}$ admits the structure of a commutative ring, then the module category admits a symmetric monoidal tensor product. Note also that there is a map $R \rightarrow R^{\prime}$ of associative ring spectra. If $R$ is concentrated over $N$ then this is a weak equivalence, and the module categories are Quillen equivalent.

If we assume that $R$ is a ring $G$-spectrum over $N$, then we obtain a ring $G / N$-spectrum $R^{N} \simeq\left(R^{\prime}\right)^{N} \simeq \Phi^{N} R$ which is strictly commutative if $R$ is, and $R \simeq \inf \Phi^{N} R \wedge \widetilde{E}[\nsupseteq$ $N]$. One way to reach this setting is to start with a ring $G / N$-spectrum $\bar{R}$ and then define $R=\inf \bar{R} \wedge \widetilde{E}[\nsupseteq N]$, noting that $R \simeq R^{\prime}$ in this case (and that $R$ need not be strictly commutative).

Theorem 7.1 If $R$ is a ring $G$-spectrum concentrated over $N$ then the fixed-point adjunction gives a Quillen equivalence

$$
\mathbb{C}_{0}=R-\text { mod-G-spectra } \simeq R^{N}-\bmod -G / N-\text { spectra } \simeq \mathbb{C}_{2}
$$

and these are also Quillen equivalent to $\mathbb{C}_{1}=L_{\Phi^{N}}(R$-module- $G$-spectra $)$.

Remark 7.2 (i) If $X$ is an $R$-module, then $X$ is a retract of $R \wedge X$, so that if $R$ lies over $N$ so too do its modules.

(ii) If $X$ lies over $N$ then $X^{N} \simeq \Phi^{N} X$. Accordingly $R^{N} \simeq \Phi^{N} R$ and Lewis-May fixed points may be replaced by geometric fixed points throughout.

Proof By the discussion in Section 4.1 we need only check that the cells generating the two module categories correspond.

Indicating the image of a subgroup in $G / N$ by a bar, we need only remark that the extensions of the cells $R^{N} \wedge \bar{G} / \bar{H}_{+}$which generate the category of $R^{N}$-module $\bar{G}-$ spectra generate $R$-module $G$-spectra. This is because the cells $G / H_{+} \wedge R$ with $H$ not containing $N$ are contractible.

For the final statement we note that since $R$ is concentrated over $N, R \simeq R \wedge \widetilde{E}[\nsupseteq N]$ so that weak equivalences coincide with weak equivalences over $N$. This means that the $\widetilde{E}[\nsupseteq N]$-Bousfield localization of $R$-module- $G$-spectra is a Quillen equivalence.

\section{Eilenberg-Moore examples}

An example of rather a different character is given by taking a $G / N$-equivariant ring spectrum $\bar{R}$ and an $N$-free $G$-space $E$ and then taking $R=F\left(E_{+}, \inf \bar{R}\right)$, so that $R^{N}=F\left(E / N_{+}, \bar{R}\right)$. We shall concentrate on $N=G$ with $E=E G$. Our focus is on the case $\bar{R}=H k$ for a commutative ring $k$, but we also comment on $\bar{R}=S^{0}$. 
Turning to the first example of the above type we take $N=G$ and

$$
R=F\left(E G_{+}, \inf H k\right) \simeq F\left(E G_{+}, H \underline{k}\right) \quad \text { so that } \quad R^{G}=F\left(B G_{+}, H k\right),
$$

where $\underline{k}$ is the Mackey functor constant at $k$. In general we write $C^{*}(X ; k)=$ $F(X, H k)$ since this is a spectrum whose homotopy is $H^{*}(X ; k)$. Thus $R=$ $C^{*}(E G ; k)$ and $R^{G}=C^{*}(B G ; k)$; we will usually not mention the coefficients explicitly.

Omitting the coefficients $k$ from the notation, we consider the following adjunction:

$$
\Psi^{G}: C^{*}(E G)-\bmod -G-\text { spectra } \longleftrightarrow C^{*}(B G)-\bmod -\text { spectra }: C^{*}(E G) \otimes_{C^{*}(B G)}(\cdot)
$$

Because of existing machinery, we will not use the methods of Section 4.3, but instead discuss directly for which $G$-spaces $X$ the unit of the adjunction is an equivalence for the $C^{*}(E G)$-module $C^{*}(E G \times X)$. Since $C^{*}(E G \times X) \simeq C^{*}(E G) \wedge D\left(X_{+}\right)$when $X$ is a finite complex, discussion of modules of this form will in particular include the generators $C^{*}(E G) \wedge G / H_{+}$. Note that if $Y$ is a free $G$-space (such as $E G \times X$ ), we have $C^{*}(Y)^{G} \simeq C^{*}(Y / G)$ so that the counit here,

$$
C^{*}(E G) \otimes_{C^{*}(B G)} C^{*}(Y / G) \rightarrow C^{*}(Y),
$$

is an embodiment of the Eilenberg-Moore spectral sequence for the fibration

$$
X \longrightarrow E G \times_{G} X \longrightarrow E G \times_{G} *=B G .
$$

When the Eilenberg-Moore spectral sequence converges, $k \otimes_{C^{*}(B G)} C^{*}(Y / G) \simeq$ $C^{*}(Y)$, so the counit is an equivalence.

Corollary 8.1 If $G$ is connected or if $\pi_{0} G$ is a $p$-group and $p^{N}=0$ on $k$ then we have a Quillen equivalence

$$
C^{*}(E G)-\bmod -G-\text { spectra } \simeq C^{*}(B G)-\bmod -\text { spectra. }
$$

Proof Under the given hypotheses, the Eilenberg-Moore spectral sequence is convergent, so that $k \otimes_{C^{*}(B G)} C^{*}(Y / G) \simeq C^{*}(Y)$ for any free $G$-space $Y$; see Dwyer [4] and Mandell [20]. Alternatively, since we have Thom isomorphisms, we may argue by Lemmas 4.5 and 4.6 that the category of $R$-modules is strongly monogenic.

In either case, the counit is an equivalence for a set of small generators, so it follows from the cellularization principle that the adjunction is a Quillen equivalence.

To highlight the significance of this result we give two examples where we do not obtain an equivalence. 
Example 8.2 If $G$ is finite and we take rational coefficients, then the cofibration $E G_{+} \rightarrow S^{0} \rightarrow \widetilde{E} G$ splits and we have a rational equivalence $C^{*}(E G) \simeq E G_{+}$. Accordingly, $C^{*}(E G)$ is free and

$$
C^{*}(E G)-\bmod -G-\text { spectra } \simeq G-\text { free }-S_{\mathbb{Q}}^{0}-\bmod -G-\text { spectra } \simeq \mathbb{Q} G-\bmod .
$$

On the other hand,

$$
S_{\mathbb{Q}}^{0} \text {-mod-spectra } \simeq \mathbb{Q} \text {-mod. }
$$

Note that the same conclusion was reached in Section 6.1. In any case, the category of $\mathbb{Q} G$-modules is not equivalent to the category of $\mathbb{Q}$-modules unless $G$ is trivial.

Example 8.3 Despite the truth of the Segal conjecture, the case with coefficients in a sphere is not so well behaved. Here we take $R=D E G_{+}=F\left(E G_{+}, S^{0}\right)$ with $N=G$ and $R^{G}=D B G_{+}=F\left(B G_{+}, S^{0}\right)$. The adjunction then takes the form

$$
\Psi^{G}: D E G_{+}-\bmod -G-\text { spectra } \longleftrightarrow D B G_{+}-\bmod -\text { spectra }: D E G_{+} \otimes_{D B G_{+}}(\cdot) .
$$

Again this is sometimes a Quillen equivalence and sometimes not. If we have rational coefficients, then $S^{0} \rightarrow H \mathbb{Q}$ is a nonequivariant equivalence, so we are back in the Eilenberg-Moore situation, where we know it is a Quillen equivalence if $G$ is a torus, but not if $G$ is a nontrivial finite group.

Carlsson's Theorem [1] implies (see work by the first author in [6] and jointly with May in [9]) that when $X$ is a based finite complex, $D\left(E G_{+} \wedge X\right) \simeq(D X)_{I}^{\wedge}$ where $I$ is the augmentation ideal of the Burnside ring. In particular, if $G$ is a $p$-group and we take coefficients in the $p$-adic sphere we have

$$
R=F\left(E G_{+},\left(S^{0}\right)_{p}^{\wedge}\right) \simeq\left(\left(S^{0}\right)_{p}^{\wedge}\right)_{I}^{\wedge} \simeq\left(S^{0}\right)_{p}^{\wedge}
$$

since some power of $I$ lies in the ideal $(p)$. Accordingly this puts us back in the situation of Section 6.

Note that since rationalization commutes with tensor products, we have

$$
\left(R \otimes_{R^{G}} M\right)_{\mathbb{Q}} \simeq R_{\mathbb{Q}} \otimes_{\left(R_{\mathbb{Q}}\right)^{G}} M_{\mathbb{Q}} .
$$

From our analysis of the rational case in Section 6 we see that we do not obtain a Quillen equivalence when $R=F\left(E G_{+},\left(S^{0}\right)_{p}^{\wedge}\right)$.

\section{The ring spectrum $D E \mathcal{F}_{+}$}

In this section we work rationally, and consider the special case when $G$ is a torus. 
One example of importance in the study of rational spectra is the counterpart of the Eilenberg-Moore example for almost free spaces (ie, spaces whose isotropy groups are all finite). Thus we let $\mathcal{F}$ denote the family of finite subgroups of $G$, and we recall the splitting theorem from the first author [7]

$$
E \mathcal{F}_{+} \simeq \prod_{F} E\langle F\rangle
$$

where the product is over finite subgroups $F$.

Taking rational duals, we find

$$
R=D E \mathcal{F}_{+} \simeq \prod_{F} D E\langle F\rangle \quad \text { and } \quad R^{G}=D\left(E \mathcal{F}_{+}\right)^{G} \simeq \prod_{F \in \mathcal{F}} D\left(B(G / F)_{+}\right) .
$$

Accordingly, this is really a question of assembling the information from the different finite subgroups. Since $G$ is abelian we have $E\langle H\rangle=E G / H_{+} \wedge \widetilde{E}[\nsupseteq H]$, so the difference between $E G / H_{+}$and $E\langle H\rangle$ is easy to describe. When $H$ is also finite and coefficients are rational, this simplifies further.

Lemma 9.1 With $R=D E \mathcal{F}_{+}$, provided $G$ is a torus and we use rational coefficients, the spheres of complex representations are $R$-built from $S^{0}$.

Proof We show that for any complex representation $V$ the suspension $S^{V} \wedge D E \mathcal{F}_{+}$ is a finite wedge of retracts of integer suspensions of $D E \mathcal{F}_{+}$.

Since $S^{V} \wedge D E \mathcal{F}_{+} \simeq D\left(S^{-V} \wedge E \mathcal{F}_{+}\right)$, it suffices to deal with the undualized form.

Now suppose we are given a complex representation $V$. Since we are working over the rationals, the classical Thom isomorphism gives an equivalence

$$
S^{V} \wedge E\langle F\rangle \simeq S^{\left|V^{F}\right|} \wedge E\langle F\rangle
$$

for each finite subgroup $F$.

Finally, we divide the finite subgroups into sets according to $\operatorname{dim}_{\mathbb{C}}\left(V^{F}\right)$. Of course there are only finitely many of these, and we may apply the corresponding orthogonal idempotents to consider the sets separately. For subgroups $F$ with $\operatorname{dim}_{\mathbb{C}}\left(V^{F}\right)=k$, the suspension is the same in each case, so that taking products over these subgroups, we find

$$
\prod_{F} D\left(E\langle F\rangle \wedge S^{V} \wedge X\right) \simeq S^{-k} \wedge \prod_{F} D(E\langle F\rangle \wedge X)
$$

as required. 
The argument of Lemma 4.4 shows that the category of $D E \mathcal{F}_{+}-$modules is strongly monogenic.

Corollary 9.2 With $R=D E \mathcal{F}_{+}, G$ a torus and rational coefficients we have an equivalence

$$
D E \mathcal{F}_{+}-\bmod -G-\text { spectra } \simeq \prod_{F} C^{*}(B G / F)-\bmod -\text { spectra } .
$$

Remark 9.3 We note that we can apply idempotents to take the factor with $F=1$ and recover the Eilenberg-Moore example of Section 8 in the case that the ambient group is a torus and the coefficients are rational.

\section{References}

[1] G Carlsson, Equivariant stable homotopy and Segal's Burnside ring conjecture, Ann. of Math. 120 (1984) 189-224 MR763905

[2] B Day, On closed categories of functors, from: "Reports of the Midwest Category Seminar, IV", Lecture Notes in Mathematics 137, Springer, Berlin (1970) 1-38 MR0272852

[3] A W M Dress, Contributions to the theory of induced representations, from: "Algebraic $K$-theory, II: "Classical" algebraic $K$-theory and connections with arithmetic", (H Bass, editor), Lecture Notes in Math. 342, Springer, Berlin (1973) 183-240 MR0384917

[4] W G Dwyer, Strong convergence of the Eilenberg-Moore spectral sequence, Topology 13 (1974) 255-265 MR0394663

[5] A D Elmendorf, I Kriz, M A Mandell, J P May, Rings, modules, and algebras in stable homotopy theory, Mathematical Surveys and Monographs 47, Amer. Math. Soc. (1997) MR1417719

[6] J P C Greenlees, Equivariant functional duals and completions at ideals of the Burnside ring, Bull. London Math. Soc. 23 (1991) 163-168 MR1122904

[7] J P C Greenlees, A rational splitting theorem for the universal space for almost free actions, Bull. London Math. Soc. 28 (1996) 183-189 MR1367167

[8] J P C Greenlees, Rational $S^{1}$-equivariant stable homotopy theory, Mem. Amer. Math. Soc. 661, Amer. Math. Soc. (1999) MR1483831

[9] J P C Greenlees, J P May, Completions of G-spectra at ideals of the Burnside ring, from: "Adams Memorial Symposium on Algebraic Topology, 2", (N Ray, G Walker, editors), London Math. Soc. Lecture Note Ser. 176, Cambridge Univ. Press (1992) 145-178 MR1232204

[10] J P C Greenlees, J P May, Some remarks on the structure of Mackey functors, Proc. Amer. Math. Soc. 115 (1992) 237-243 MR1076574 
[11] J P C Greenlees, J P May, Generalized Tate cohomology, Mem. Amer. Math. Soc. 543, Amer. Math. Soc. (1995) MR1230773

[12] J P C Greenlees, B Shipley, An algebraic model for free rational $G$-spectra, to appear in Bull London Math. Soc arXiv:1101.4818

[13] J P C Greenlees, B Shipley, An algebraic model for rational torus-equivariant stable homotopy theory arXiv:1101.2511v3

[14] J P C Greenlees, B Shipley, An algebraic model for rational torus-equivariant stable homotopy theory, new version in preparation

[15] J P C Greenlees, B Shipley, The cellularization principle for Quillen adjunctions arXiv: 1301.5583

[16] M Hill, M Hopkins, Equivariant multiplicative closure arXiv:1303.4479

[17] PS Hirschhorn, Model categories and their localizations, Mathematical Surveys and Monographs 99, Amer. Math. Soc. (2003) MR1944041

[18] L G Lewis, Jr, The category of Mackey functors for a compact Lie group, from: "Group representations: cohomology, group actions and topology", (A Adem, J Carlson, S Priddy, P Webb, editors), Proc. Sympos. Pure Math. 63, Amer. Math. Soc. (1998) 301-354 MR1603183

[19] L G Lewis, Jr, J P May, M Steinberger, J E McClure, Equivariant stable homotopy theory, Lecture Notes in Mathematics 1213, Springer, Berlin (1986) MR866482

[20] M A Mandell, $E_{\infty}$ algebras and p-adic homotopy theory, Topology 40 (2001) 43-94 MR1791268

[21] M A Mandell, J P May, Equivariant orthogonal spectra and $S$-modules, Mem. Amer. Math. Soc. 755, Amer. Math. Soc. (2002) MR1922205

[22] J E McClure, $E_{\infty}$-ring structures for Tate spectra, Proc. Amer. Math. Soc. 124 (1996) 1917-1922 MR1307549

[23] S Schwede, B Shipley, Equivalences of monoidal model categories, Algebr. Geom. Topol. 3 (2003) 287-334 MR1997322

[24] S Schwede, B Shipley, Stable model categories are categories of modules, Topology 42 (2003) 103-153 MR1928647

[25] J Ullman, Tambara functors and commutative ring spectra arXiv: 1304.4912

Department of Pure Mathematics, University of Sheffield The Hicks Building, Sheffield S3 7RH, UK

Department of Mathematics, Statistics and Computer Science, University of Illinois at Chicago 508 SEO (m/c 249), 851 South Morgan Street, Chicago, IL 60607-7045, USA

j.greenlees@sheffield.ac.uk, bshipley@math.uic.edu

Received: 16 February $2013 \quad$ Revised: 23 October 2013 
